\title{
DBT-A reduces self-harming behaviours by improving feelings of hopelessness
}

\author{
By Jessica K. Edwards
}

In 2014, a randomized controlled trial conducted by Lars Mehlum and colleagues showed that a comparatively brief course of Dialectical Behaviour Therapy adapted for adolescents (DBT-A) is superior to enhanced usual care (EUC) in reducing self-harming behaviour, suicidal ideation, and depressive symptoms, 1 and that DBT-A remains superior in reducing self-harming behaviours up to 1 year after treatment.2 Now, Lars Mehlum and colleagues have completed a prospective 3-year followup study, which showed that DBT-A has enduring effects in terms of reducing self-harm frequency in adolescents compared to EUC. A substantial proportion of the effect of DBT-A on self-harm over the longterm was mediated by a reduction in the participants' experience of hopelessness during the trial treatment. The researchers also found that receiving $>3$ months follow-up treatment in the first year after completion of the trial treatment was associated with further enhanced outcomes in patients who had received DBT-A.

How a reduction in the levels of hopelessness during DBT-A might mediate a long-term reduction in selfharming behaviours is unclear. Mehlum and colleagues highlight, however, that DBT-A includes several interventions to address and treat hopelessness and promote dialectical thinking to help patients change their polarized perceptions of self and others. Going forward, the researchers propose that therapeutic interventions aiming to reduce self-harm in adolescents should focus on hopelessness and other cognitive or emotional factors that might otherwise prevent recovery.

Mehlum, L. (2019), Long term effectiveness of dialectical behaviour therapy versus enhanced usual care for adolescents with self-harming and suicidal behaviour. J. Child Psychol. Psychiatr. doi:10.1111/jcpp.13077

\section{References}

${ }^{1}$ Mehlum, L., Tormoen, A.J., Ramberg, M., Haga, E., Diep, L.M., Laberg, S. and Groholt, B. (2014). Dialectical behaviour therapy for adolescents with repeated suicidal and self-harming behaviour: A randomized trial. J. Am. Acad. Child Adolesc. Psychiatry, 53, 1082- 1091. doi: 10.1016/j. jaac.2014.07.003.

${ }^{2}$ Mehlum, L., Ramberg, M., Tormoen, A.J., Haga, E., Diep, L.M., Stanley, B.H. and Groholt, B. (2016). Dialectical behaviour therapy compared with enhanced usual care for adolescents with repeated suicidal and self-harming behaviour: Outcomes over a one-year follow-up. J. Am. Acad. Child Adolesc. Psychiatry, 55, 295-300. doi: 10.1016/j. jaac.2016.01.005.

\section{Glossary}

Dialectical Behaviour Therapy (DBT): A multicomponent cognitive-behavioural treatment, in which the patient learns to manage difficult emotions by experiencing, recognizing and accepting them. DBT therapies use a balance of acceptance (accepting yourself as you are) and change (making positive changes in your life) techniques. Once the patient has learnt to accept and regulate emotions, they are then more able to change a harmful behaviour, such as self-harming. In general, DBT includes individual psychotherapy, family group skills training, telephone coaching, and therapist team consultations. 